\title{
Portable Electron Microscopy and Microanalysis in Extreme Environments
}

\author{
Christopher S. Own ${ }^{1}$, Matthew F. Murfitt ${ }^{1}$, Lawrence S. Own ${ }^{1}$, Jesse Cushing ${ }^{1}$, James Martinez ${ }^{2}$, Katherine \\ Thomas-Keprta ${ }^{2}$, Donald R. Pettit ${ }^{3}$ \\ ${ }^{1 .}$ Voxa, Seattle, WA, USA. \\ 2. Jacobs/JETS NASA Johnson Space Center, Houston, TX, USA. \\ ${ }^{3 .}$ NASA Johnson Space Center, Houston, TX, USA.
}

Electron microscopy (EM) is a highly attractive tool for many applications due to its unique blend of strong optical scattering, high native resolution, large depth of focus, and variety of signals including characteristic Xray emission, enabling high-magnification structural imaging and chemical analysis. Despite high optical performance and versatility supporting a wide variety of industries from basic science research to industrial process monitoring, EM has through its $~ 100$-year history been widely regarded as a high-end tool with limited reach outside the laboratory, in particular due to inherent complexity and need for vacuum. Making EM accessible outside constrained laboratory environments will bring EM's performance and versatility to a much broader range of scientific and engineering endeavors.

EM's are traditionally housed in centers at universities and large research institutions where those without access to on-site EM typically send samples to be imaged, at great expense and often with delay of weeks to months for complex analyses. For current field work, samples must be sent back to a facility where they can experience chemical or morphological changes over time and/or may be damaged in transit and cannot be reliably analyzed. On-site EM - defined here as EM for immediate portable use in remote or extreme environments at or near the site of sample collection - has historically been impractical.

Mochii $^{\mathrm{TM}}$ is a portable commercial scanning EM developed by the coauthors at Voxa in Seattle, WA to address the need for EM outside the laboratory (Fig. 1) [1]. This tiny low voltage microscope, which fits in the overhead bin of an airplane, has features that bring accessible and on-demand EM imaging to new applications previously hindered by size, complexity, and cost. Among these features are hand-carryable form-factor and low power consumption $(0.25 \mathrm{~m}$ tall, $<12 \mathrm{~kg},<80 \mathrm{~W})$, user-friendly native wireless tablet interface, multi-user and remote capabilities, an integrated metal evaporator for easy sample preparation, and optional energy-dispersive X-ray analyzer for chemical identification [2]. The cost to own and operate a Mochii microscope is a fraction of the cost of typical EM's possessing similar imaging performance due to its low power consumption, simple design, and commoditized user-replaceable consumables. At the meeting we will report on use of EM and considerations for use in extreme field environments, such as outdoors under battery power, on moving vehicles such as ocean vessels, and perhaps the most extreme of environments: space (low earth orbit (LEO) and deep space).

We are in process to prepare Mochii for manned spaceflight. Ground-based versions of EM's have been essential in NASA research for many years. In mineralogy and petrology, for example, EM is used to understand the origin and evolution of the solar system, particularly rocky bodies, through detailed study of asteroidal and cometary samples. In microbiology, EM has been used to visualize the architecture of tissues and cells to understand effects of microgravity on living things. In engineering/materials science it has been used to characterize particulate debris in air and water samples from vehicle systems, determine pore sizes in ceramics/catalysts, understand the nature of fibers, determine the composition and morphology of new and existing materials, and characterize the micro-textures of vapor deposited films. All of these, covering many of NASA's core inquiries, will be significantly enhanced by on-site analyses including on manned vehicles and also robotic missions, and enable NASA to address a current blind spot in its detection and analysis toolset. Mochii will demonstrate real-time, on-site imaging and compositional measurements aboard the International Space Station (ISS), accelerating answers to many scientific inquiries and mission decisions. 
An example highlighting some advantages of EM in space is in the study of microgravity crystallization dynamics [3-4], shown in Fig. 2. $\mathrm{NaCl}$ is optically transparent and its fine faceted surfaces are difficult to clearly image using light microscopy. EM elucidates these tiny structures and amplifies sensitivity to differences in experimental conditions during crystal growth. While these samples were imaged post-mission using Mochii EM on Earth, on-site EM coupled with elemental microanalysis will enable these and other experiments to be conducted on-vehicle, including those in which irreversible phase or chemical transitions occur in the presence of gravity or atmospheric conditions. Such studies would be impossible if re-entry back to Earth is required before analysis.

On-vehicle crew time is extremely precious, and innovations in this area are needed to facilitate effective use on ISS. Collaborative easy-to-use and responsive multi-user interfaces enable scientists on the ground to remotely operate the system while minimizing the impact to crew time. Another key innovation is a quick-release optical column cartridge system that enables instant removal and insertion of factory pre-aligned full column cartridges [5]. This quick change between prepackaged optical configurations (e.g., analytical vs high resolution) has minimal impact to the end user's cognitive and service load and supports more analyses without additional overhead.

On-site EM represents a significant paradigm shift in microscopy, taking high-resolution analytical microscopy and microanalysis out of the lab and into new environments, and increasing access to important scientific phenomena at the micro- and nanoscale [6].

\section{References:}

[1] CS Own, et al, Microscopy \& Microanalysis (2015), p. 57.

[2] CS Own and MF Murfitt, US Patent No. 9,564,291 (2017).

[3] P Fontana, D Pettit, S Cristoforetti, Journal of Crystal Growth 428 (2015), p. 80.

[4] P Fontana, J Schefer, D Pettit, Journal of Crystal Growth 324 (2011), p. 207.

[5] CS Own, US Patent App No. 14/607,079 (2015).

[6] This work was supported by Voxa, NASA, and Jacobs.

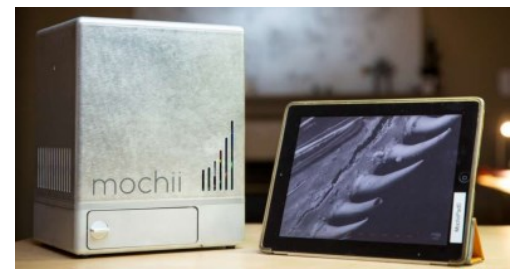

Figure 1. (left) Voxa's Mochii ${ }^{\mathrm{TM}}$ Electron Microscope (left) with iPad controller (right) showing a high resolution image of the head of a wheat plant [1].

Figure 2. (below) (a) $\mathrm{NaCl}$ polycrystals formed in earth gravity by evaporation, and (b) Large $\mathrm{NaCl}$ monocrystals formed in microgravity by evaporation. Large stable monolithic crystals form from a small number of nucleation sites in microgravity. Both imaged in Mochii ${ }^{\mathrm{TM}} \mathrm{EM}$ on earth.
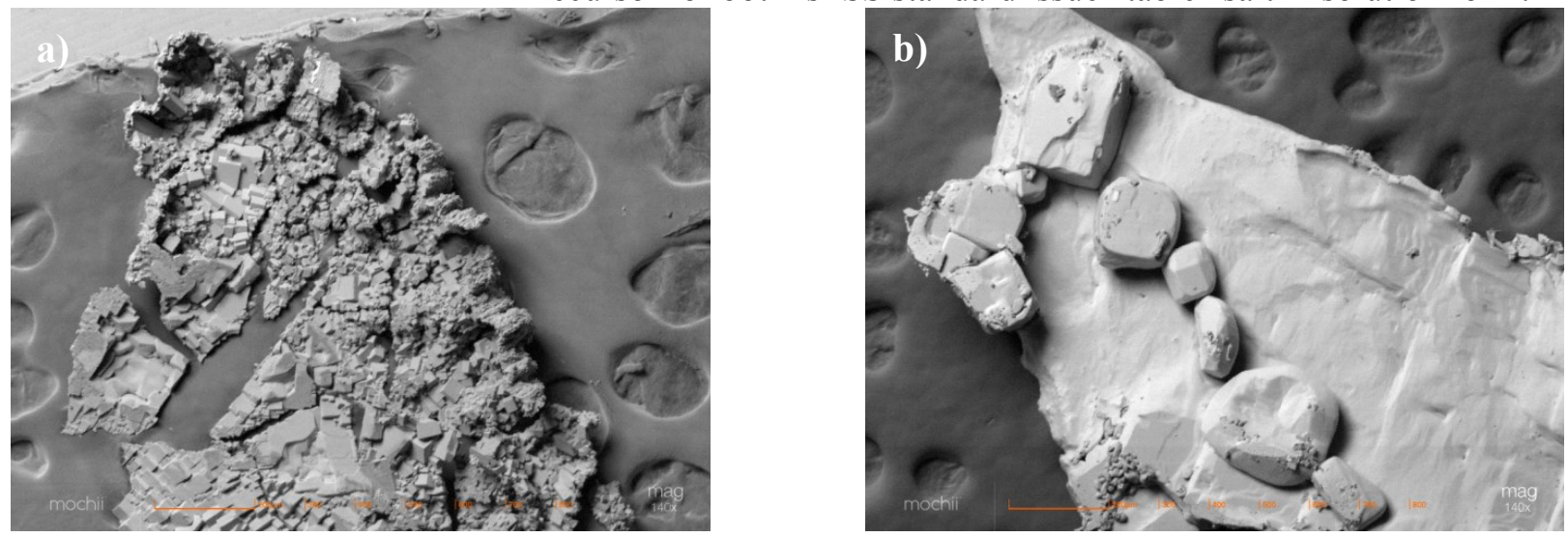